\title{
Control of the perpendicular magnetic anisotropy of FePd films via Pd capping deposition
}

\author{
C. Clavero, ${ }^{1,2, a)}$ J. R. Skuza, ${ }^{3}$ Y. Choi, ${ }^{4}$ D. Haskel, ${ }^{5}$ J. M. García-Martín, ${ }^{2}$ A. Cebollada, ${ }^{2}$ \\ and R. A. Lukaszew 1,3 \\ ${ }^{1}$ Department of Applied Science, College of William \& Mary, Williamsburg, Virginia 23187, USA \\ ${ }^{2}$ Instituto de Microelectrónica de Madrid, IMM (CNM-CSIC), Tres Cantos, Madrid 28760, Spain \\ ${ }^{3}$ Department of Physics, College of William \& Mary, Williamsburg, Virginia 23187, USA \\ ${ }^{4}$ Materials Science Division, Argonne National Laboratory, Argonne, Illinois 60439, USA \\ ${ }^{5}$ Advanced Photon Source, Argonne National Laboratory, Argonne, Illinois 60439, USA
}

(Received 3 February 2008; accepted 7 April 2008; published online 21 April 2008)

\begin{abstract}
We have investigated the influence of two capping layers $(\mathrm{MgO}$ and $\mathrm{Pd})$ on the magnetic anisotropy of highly anisotropic $L 1_{0} \mathrm{FePd}$ films. While we mainly found perpendicular magnetic anisotropy in $\mathrm{MgO}$ capped films, we observed that the Pd capping layer induces formation of an additional new phase near the FePd/Pd interface that exhibits in-plane magnetic anisotropy. The combined effect of these two anisotropies results in global canted magnetic anisotropy. Thus, our findings illustrate a mechanism to influence the magnetic anisotropy in FePd highly ordered alloys via adequate choice of capping layer materials. (C) 2008 American Institute of Physics. [DOI: 10.1063/1.2916712]
\end{abstract}

It has been shown that the magnetic properties of materials are strongly affected by size reduction and interfacing with other materials. ${ }^{1}$ This is particularly important for materials that could potentially yield increased data storage densities, such as FePt and FePd, whose highly anisotropic chemically ordered $L 1_{0}$ phase can withstand further size reduction pushing the superparamagnetic limit. ${ }^{2}$ When these magnetic alloys are in thin film form, parameters such as substrate-film lattice mismatch and deposition conditions can affect the degree of chemical order achievable. In addition, capping layers can also have a significant effect on magnetic properties. ${ }^{3}$ Here, we present our structural studies correlated with magnetic characterizations carried out on FePd films grown under identical conditions but with different capping layers (i.e., $\mathrm{Pd}$ and $\mathrm{MgO}$ ), thus, providing useful information about the capping layer influence on the magnetic anisotropy.

FePd films were cosputtered on (001) $\mathrm{MgO}$ substrates in ultrahigh vacuum conditions (background pressure 5 $\times 10^{-9}$ Torr). It has already been shown that the $9 \%$ mismatch between the $\mathrm{FePd}$ and $\mathrm{MgO}$ lattices promotes three-dimensional (3D) growth mode. ${ }^{4}$ In addition, optimal sputtering conditions to achieve films with adequate stoichiometry leading to $L 1_{0}$ order have been determined. In particular, it was found that the optimal substrate temperature for long-range $L 1_{0}$ order is $450{ }^{\circ} \mathrm{C}$, with a strong correlation between film thickness and average roughness for films grown at this temperature. ${ }^{4}$ In order to investigate the effect of the capping layer type on the magnetic anisotropy, two series of FePd films deposited at $450{ }^{\circ} \mathrm{C}$ with varying thicknesses $(3-20 \mathrm{~nm})$ were capped with two different materials. The $3 \mathrm{~nm}$ thick capping layers were deposited at room temperature on each series of FePd films and consisted of $\mathrm{MgO}$ and $\mathrm{Pd}$, respectively. $\mathrm{Pd}$ is magnetically polarizable and promotes the connection between magnetic regions ${ }^{3}$ while no significant effect is expected for the $\mathrm{MgO}$ capped samples as was observed.

The structures were probed with $\mathrm{x}$-ray diffraction (XRD) using a standard four-circle diffractometer with $\mathrm{Cu} K \alpha$ radia-

\footnotetext{
a)Electronic mail: cclavero@wm.edu.
}

tion $(\lambda=1.5418 \AA)$. The presence of chemically ordered $L 1_{0}$ phase is evidenced by the appearance of specific diffraction peaks: the (002) fundamental reflection, and the so-called superlattice reflection (001), corresponding to the doubled periodicity in the unit cell. The $L 1_{0}$ ordered phase was clearly achieved in the $\mathrm{MgO}$ capped samples, as shown in Fig. 1(a), where the bulk momentum transfer $\left(q_{z}\right)$ positions of the (001) and (002) reflections are indicated by dashed vertical lines. The two peaks centered at the same position correspond to two different grain sizes. ${ }^{4}$ In addition, a small amount of FePd disordered alloy was also detected in the thicker film. Regarding the Pd capped system [Fig. 1(b)], we observed the Pd reflection at $q_{z}=0.323 \mathrm{~nm}^{-1}$ and a reduction in the amount of $L 1_{0}$ phase for all FePd thicknesses, concomitant with the appearance of a secondary FePd reflection at smaller scattering angles. This reflection corresponds either to a phase with a smaller degree of ordering or a Pd rich $\mathrm{FePd}$ phase. It is worth noticing that this is the only phase found in the thinner Pd capped films (thickness below $4 \mathrm{~nm}$ ),

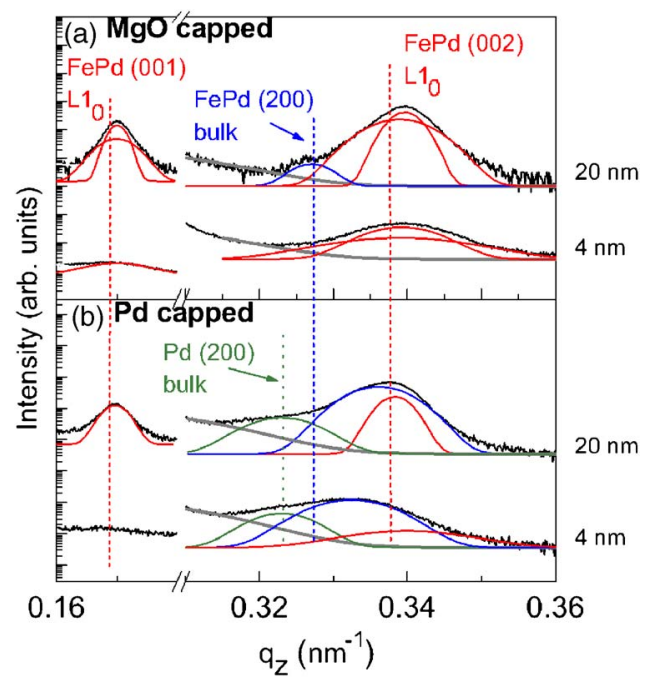

FIG. 1. (Color online) XRD scans for 4 and $20 \mathrm{~nm}$ thick FePd films capped with (a) $\mathrm{MgO}$ and (b) Pd. The position of the bulk FePd(001), FePd(002), FePd(200), and $\operatorname{Pd}(200)$ reflections are marked by vertical lines. The thick grey lines represent the $\mathrm{MgO}$ substrate contribution. 


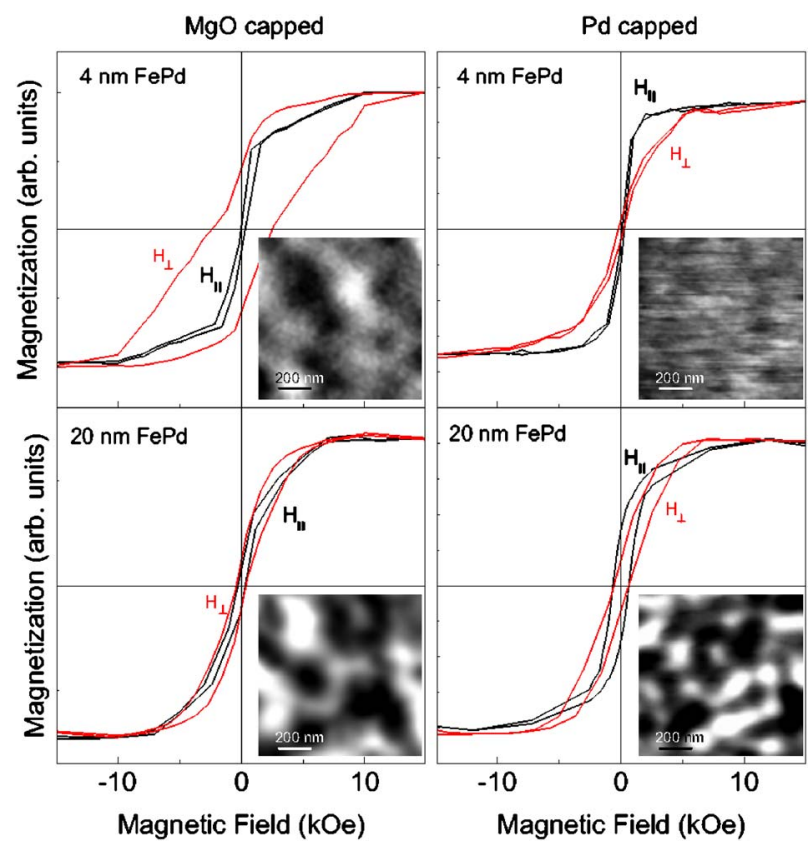

FIG. 2. (Color online) Perpendicular $(H \perp)$ and in-plane $(H \|)$ SQUID loops for the 4 and $20 \mathrm{~nm}$ thick FePd films capped with $\mathrm{MgO}$ and Pd. The insets show MFM images of the samples (lateral size: $1 \mu \mathrm{m}$ ).

whereas, thicker FePd films had increasing amounts of $L 1_{0}$. This suggests that the new phase is formed at the topmost part of the films, most likely during the sputter deposition of the Pd capping layer.

The magnetic anisotropy was investigated in both systems measuring hysteresis loops at room temperature with a superconducting quantum interference device magnetometer (SQUID). Measurements on the 4 and $20 \mathrm{~nm}$ thick FePd films capped with $\mathrm{Pd}$ or $\mathrm{MgO}$ are shown in Fig. 2. We observe perpendicular magnetic anisotropy corresponding to the $L 1_{0}$ ordered phase in the case of the $4 \mathrm{~nm}$ FePd film capped with $\mathrm{MgO}$, which is demonstrated by large perpendicular coercivity and remanence, together with large inplane saturation field. On the other hand, a very different behavior is found in the $4 \mathrm{~nm} \mathrm{FePd}$ film capped with $\mathrm{Pd}$ (completely consisting of the disordered phase mentioned above, as shown by XRD). Here, only the in-plane anisotropy is observed, demonstrated by the disappearance of perpendicular coercivity and the drastic reduction of the inplane saturation field. Regarding the thicker films $(20 \mathrm{~nm}$ $\mathrm{FePd}$ ), the $\mathrm{MgO}$ capped sample shows a strong reduction of the coercive field, evidencing the continuous layer nature of the films in sharp contrast with our observation in the thinner sample. ${ }^{4}$ Nevertheless, despite the demagnetizing field, perpendicular magnetic anisotropy is still observed. For the $20 \mathrm{~nm} \mathrm{FePd}$ film capped with Pd, where $L 1_{0}$ ordered and disordered phases are both present, a mixed magnetic anisotropy behavior is found as well, which is exhibiting high values of remanence in both in-plane and perpendicular directions. This evidences a global canted magnetic anisotropy.

Magnetic force microscopy (MFM) experiments were performed with the samples prepared in remanent state after a demagnetizing process in the perpendicular direction. Representative images are included as insets in Fig. 2. The black and white regions correspond to north and south magnetic poles at the surface, ascribed to magnetic domains with perpendicular magnetization pointing upwards and downwards, respectively. This is consistent with the loops already disAuthor complimentary copy. Redistribution subject to AIP cussed for $\mathrm{MgO}$ capped films and for the $20 \mathrm{~nm}$ thick Pd capped film, considering the perpendicular component of the magnetization. For the $4 \mathrm{~nm}$ thick Pd capped film, only noise was obtained in the measurement, i.e., no poles were detected, indicating that the magnetization is mainly in the plane of the sample and uniform in the scanned region.

In order to further elucidate the influence of the $\mathrm{Pd}$ capping layer on the magnetization depth profile in the FePd films, x-ray resonant magnetic scattering (XRMS) measurements were carried out at undulator beamline 4-ID-D of the advanced photon source. The x-ray energy was tuned to the $\operatorname{Pd} L_{3}$ absorption edge $\left(2 p_{3 / 2} \rightarrow 4 d\right.$ transition at $E=3.173 \mathrm{keV})$ with a $\mathrm{Si}(111)$ double crystal monochromator. A superconducting magnet, mounted on a diffractometer, provided a $H= \pm 30 \mathrm{kOe}$ field along the incident $\mathrm{x}$-ray wave vector $\mathbf{k}_{i}$ which nearly coincides with the sample's surface. The dichroic XRMS measurements were carried out by switching the helicity of circularly polarized x-rays at each scattering vector $q_{z}$. Measurements were also carried out for opposite directions of the applied field. Since helicity switching is equivalent to field reversal, this procedure allows checking for experimental artifacts. Two $14 \mathrm{~nm}$ thick FePd films, capped with $\mathrm{Pd}$ and $\mathrm{MgO}$, respectively, were measured in this fashion. X-ray specular reflectivity curves (Cu $K \alpha$ radiation) for both samples are shown in Figs. 3(a) and 3(b). A small number of wiggles are found due to the 3D growth mode observed in these films. ${ }^{4}$ The measured curves were fitted using Parratt's recursive formalism with roughness. ${ }^{5,6}$ The fits yield $\mathrm{MgO}(001) / \mathrm{MgO}$ buffer $(10 \mathrm{~nm}) / \mathrm{FePd}(14 \mathrm{~nm}) / \mathrm{Pd}(4.2 \mathrm{~nm})$ and $\mathrm{MgO}(001) / \mathrm{MgO}$ buffer $(6.5 \mathrm{~nm}) / \mathrm{FePd}(14 \mathrm{~nm}) / \mathrm{MgO}(7 \mathrm{~nm})$ for the two structures, respectively. Fitted values of rms roughness are in the $1-1.5 \mathrm{~nm}$ range for all interfaces. Fitted layer densities are within $80 \%$ of their bulk values. In order to extract information on the magnetization density of the films in the depth direction, XRMS measurements were done on the two above mentioned samples in charge-magnetic interference reflectivity mode. ${ }^{7}$ The asymmetry ratio, $\Delta R=\left(I^{+}-I^{-}\right) /\left(I^{+}\right.$ $\left.+I^{-}\right)$, where $(+,-)$denote opposite x-ray helicity, is plotted in Figs. 3(c) and 3(d) for opposite directions of the applied field as a function of $q_{z}$ for each sample. The structural parameters obtained in the fits of the specular reflectivity curves were used in the fits of the charge-magnetic specular reflectivity data, which are carried out within the distortedwave Born approximation. ${ }^{8,9}$ Imaginary parts of resonant chemical and magnetic scattering factors at the $\mathrm{Pd} L_{3}$ edge were obtained from published $\mathrm{x}$-ray absorption and $\mathrm{x}$-ray magnetic circular dichroism spectra ${ }^{10}$ while real parts were obtained through a differential Kramers-Krönig transform. The procedure uses tabulated scattering factors away from resonance for absolute normalization. 7,11,12

A good fit to the XRMS data of the $\mathrm{MgO}$ capped sample could be obtained, assuming that the entire FePd film is uniformly, magnetized as shown in Fig. 3(e). From Fig. 2, it is expected that an applied field of $30 \mathrm{kOe}$ is large enough to magnetically saturate the sample in the film plane. Conversely, a model assuming a nonuniform magnetization profile was needed to fit the data for the Pd capped samples. In this case, a good fit is obtained when the FePd films are split into two regions, the top region near the $\mathrm{FePd} / \mathrm{Pd}$ interface magnetized more strongly in-plane than the bottom region near the $\mathrm{FePd} / \mathrm{MgO}$ buffer interface. The enhancement of the in-plane magnetization density in the top part of the film license or copyright, see http://apl.aip.org/apl/copyright.jsp 

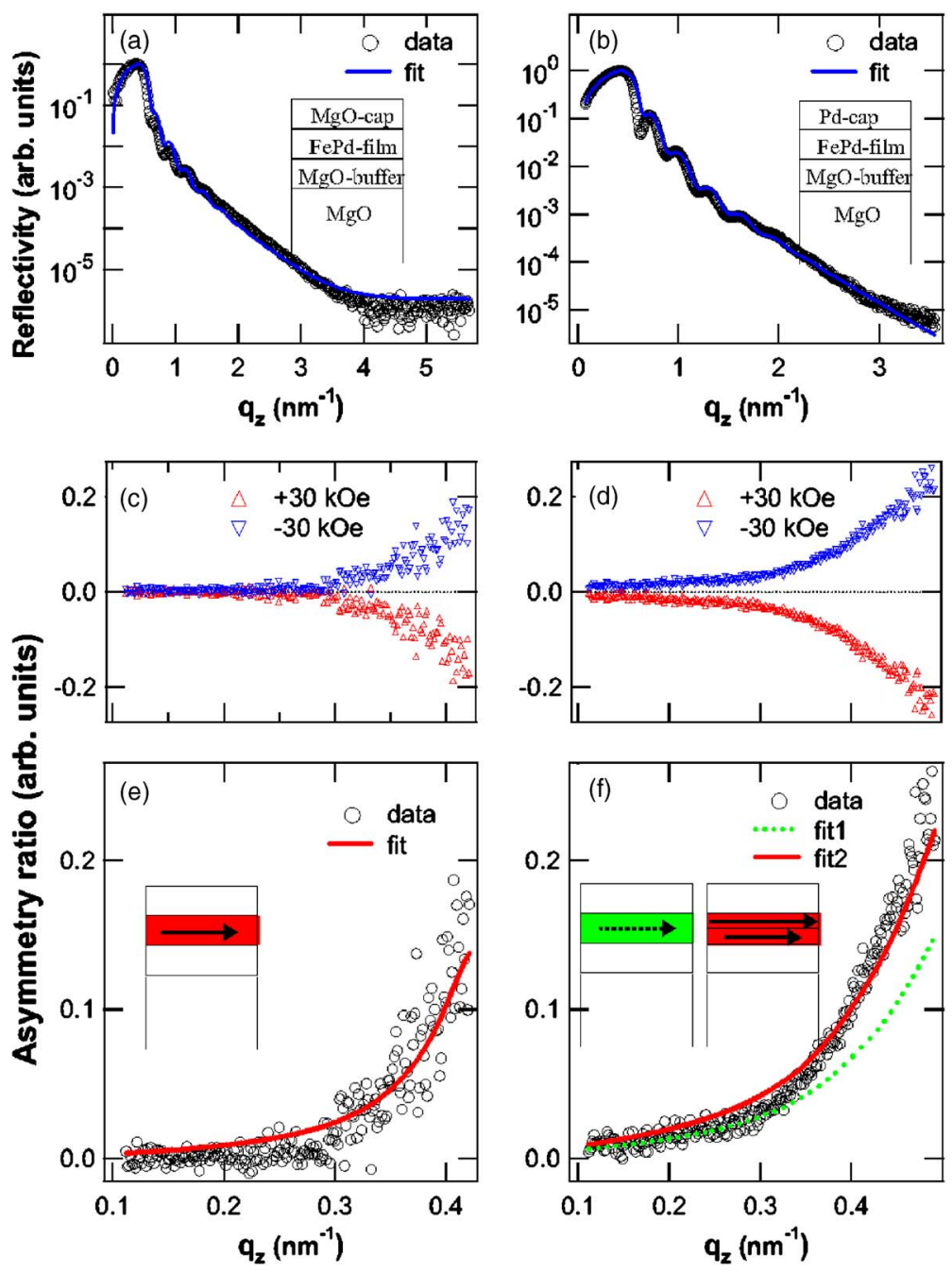

FIG. 3. (Color online) [(a) and (b)] Measured and calculated reflectivity curves with $\mathrm{Cu} K \alpha$ radiation for $14 \mathrm{~nm}$ thick FePd films capped with $\mathrm{MgO}$ and $\mathrm{Pd}$, respectively. Measured XRMS curves with two field directions for the (c) $\mathrm{MgO}$ capped sample and for the (d) Pd capped sample. [(e) and (f)] Measured and calculated XRMS curves.

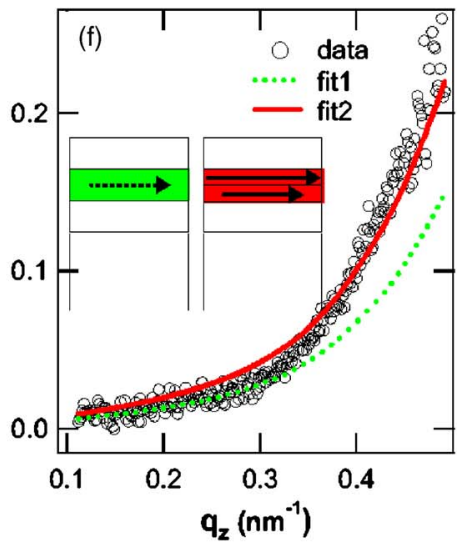

(7.8 nm thick, about half the FePd layer) is $50 \%$ relative to the bottom part in this sample and also to the $\mathrm{MgO}$ capped case. Figure 3(f) also shows that a homogeneous magnetization profile in the FePd film cannot explain the XRMS data in the Pd capped sample. Despite the limited spatial resolution $(6 \mathrm{~nm})$ in our magnetization depth profile measurements, the measurements clearly demonstrate the presence of an enhanced in-plane magnetization near the Pd capping. We note that for these small scattering angles, the XRMS asymmetry ratio is mostly sensitive to magnetization density in the plane of the film (which nearly coincides with the applied field) and hence, magnetization density along the sample's perpendicular direction results in no magnetic contrast. ${ }^{7}$

In summary, our studies show that the choice of capping material strongly affects the structural and magnetic anisotropy properties of highly ordered FePd films. In the particular case of Pd capping, XRD studies indicate the presence of a new phase in the upper portion of the FePd films, which is very likely formed during the Pd capping deposition. It was found that this new phase exhibits in-plane magnetic anisotropy, strongly modifying the global magnetic anisotropy of the films and leading to resultant canted magnetic anisotropy. Thus, our findings illustrate a mechanism to influence the magnetic anisotropy in highly ordered alloys via adequate choice of capping layer materials. This is a critical issue with important applications such as magnetic storage media.
This work was partially supported by NSF-DMR (0355171), the ACS and the Research Corporation. Work at Argonne is supported by the U.S. DOE and OS (DE-AC-02-06CH11357). The CAM (NANOMAGNET S-0505/MAT/0194), MEC (MAT2005-05524-C02-01 and NAN2004-09195-C04-01), and CSIC (200650I130) are also acknowledged.

${ }^{1}$ X. Batlle and A. Labarta, J. Phys. D: Appl. Phys. 35, R15 (2002).

${ }^{2}$ D. Weller and A. Moser, IEEE Trans. Magn. 35, 4423 (1999).

${ }^{3}$ E. Navarro, Y. Huttel, C. Clavero, A. Cebollada, and G. Armelles, Phys. Rev. B 69, 224419 (2004).

${ }^{4}$ C. Clavero, J. M. García-Martín, J. L. Costa Kramer, G. Armelles, A. Cebollada, Y. Huttel, R. A. Lukaszew, and A. J. Kellock, Phys. Rev. B 73, 174405 (2006).

${ }^{5}$ L. G. Parratt, Phys. Rev. 95, 359 (1954).

${ }^{6}$ L. Nevot and P. Croce, Rev. Phys. Appl. 15, 761 (1980).

${ }^{7}$ D. Haskel, G. Srajer, J. C. Lang, J. Pollmann, C. S. Nelson, J. S. Jiang, and S. D. Bader, Phys. Rev. Lett. 87, 207201 (2001)

${ }^{8}$ D. R. Lee, S. K. Sinha, D. Haskel, Y. Choi, J. C. Lang, S. A. Stepanov, and G. Srajer, Phys. Rev. B 68, 224409 (2003).

${ }^{9}$ S. K. Sinha, E. B. Sirota, S. Garo, and H. B. Stanley, Phys. Rev. B 38, 2297 (1988).

${ }^{10}$ J. Vogel, A. Fontaine, V. Cros, F. Petroff, J.-P. Kappler, G. Krill, A. Rogalev, and J. Goulon, Phys. Rev. B 55, 3663 (1997).

${ }^{11}$ D. T. Cromer and D. Liberman, J. Chem. Phys. 53, 1891 (1970).

${ }^{12}$ J. O. Cross, M. Newville, J. J. Rehr, L. B. Sorensen, C. E. Bouldin, G. Watson, T. Gouder, G. H. Lander, and M. I. Bell, Phys. Rev. B 58, 11215 (1998). 